\title{
SEX STEROID HORMONES AND VOLUNTARY EXERCISE IN RATS. A CORRECTION
}

\author{
S. A. ASDELL AND G. A. SPERLING \\ Animal Husbandry Department, Cornell University, Ithaca, New York, U.S.A.
}

(Received 24th October 1962)

\begin{abstract}
Summary. A result reported earlier that oestradiol sensitizes the nervous system of the male rat so that it responds to testosterone with increased motor activity could not be repeated. The male and female rat respond differently, the male only to oestradiol and the female to both oestradiol and testosterone. A few rats sometimes respond to testosterone.
\end{abstract}

Oestradiol benzoate pellets implanted beneath the skin of the gonadectomized rat cause both males and females to take large amounts of exercise in an activity drum. Testosterone propionate causes the females to take much exercise but has a small effect upon the males. Asdell, Doornenbal \& Sperling (1962) have suggested that testosterone propionate is effective in males if the implant follows one of oestradiol benzoate. Therefore it was postulated that oestrogen sensitizes the nervous system to androgen. We have been unable to repeat this work and believe that the previous result was obtained because the testosterone implant was made before the direct effect of the oestrogen had disappeared.

Eight male rats were castrated when about 45 days old. A week later, each was implanted subcutaneously with one pellet containing $1 \mathrm{mg}$ of oestradiol benzoate. All responded with increased activity and in all but one a daily average of over 10,000 rotations of the activity drum, about 7 miles, was reached. At the end of 8 weeks, when activity had decreased to normal levels, each rat was implanted with one pellet containing $1 \mathrm{mg}$ of testosterone propionate. During the following 8 weeks, no increased activity was observed in any of the rats. They were then implanted with $1-\mathrm{mg}$ oestradiol pellets and all displayed increased activity but not to the degree reached in the earlier oestrogen period. After 8 weeks, another testosterone implantation was made. Activity increased slightly in two rats only.

In an experiment designed to test the effect of testosterone level, eight male rats were castrated when about 45 days old. Two were implanted with single 1-mg testosterone propionate pellets. One showed a marked increase in activity; the other had a small response. With two l-mg pellets, one rat increased markedly and the other showed a slight response. With a single 10-mg pellet, one rat responded markedly; the second with a little increased activity. With two 10-mg pellets, one rat increased markedly and the second to a small degree. After 8 weeks, l-mg testosterone propionate pellets were 
implanted. None of the eight rats showed increased activity. None of these rats had received oestrogen implants to this time and it is clear that some rats may respond to testosterone without previous treatment. After an interval of 8 weeks, 1-mg oestradiol benzoate pellets were implanted. Four of the rats greatly increased their activity, while the other four showed smaller but quite definite responses. After a further 8 weeks, $1-\mathrm{mg}$ pellets of testosterone propionate were implanted. One rat, which had been exceptionally active to the end of the 8-week period, showed greatly increased activity (17,296 rotations per day, the equivalent of 12 miles), which is reminiscent of the result obtained in the paper cited earlier. Two other rats displayed slight increases and the remaining five none.

From this work we conclude that oestrogen does not sensitize the male rat so that it responds to testosterone with increased voluntary motor activity. The earlier report was misleading, probably because the interval between implants was too short. We are led to the conclusion mentioned (but rejected) in the earlier paper, that male and female rats differ in their response to the two sex steroids. The female responds by motor activity to oestrogen and to androgen, while, paradoxically, the male responds to oestrogen and, as a rule, only slightly, or not at all, to androgen.

\section{ACKNOWLEDGMENTS}

This work was supported by U.S.P.H.S. research grant A3299 Endo. The pellets were supplied by the Merck Institute for Therapeutic Research.

\section{REFERENCE}

Aspeli, S. A., Doornenbai., H. \& Speri.ing, G. A., (1962) Sex steroid hormones and voluntary exercise in rats. F. Reprod. Fertil. 3, 26. 